\title{
A novel tethered-sphere add-on to enhance grid turbulence
}

\author{
Richard Vonlanthen $\cdot$ Peter A. Monkewitz
}

Received: 23 September 2010/Revised: 7 March 2011/Accepted: 8 March 2011/Published online: 24 March 2011

(c) Springer-Verlag 2011

\begin{abstract}
The new turbulence generator consists of a standard uniform grid with tethered spheres attached to its nodes and is capable of producing approximately twice the turbulence energy per unit pressure drop coefficient $C_{\mathrm{p}}$ than the same bare grid without the spheres. At the same time, the Reynolds number $\operatorname{Re}_{\lambda}$ based on the Taylor microscale is also amplified by a factor of roughly 2, and the turbulence anisotropy is reduced to a constant level of $10 \%$ at all downstream distances without further flow conditioning after the grid. The new grid's simple design makes it suitable for a variety of fluid-flow facilities, in particular smaller water tunnels. Its performance in comparison with the plain grid is documented by measurements of the streamwise decay of turbulence energy and velocity spectra in the $\operatorname{Re}_{\lambda}$ range of $50-100$.
\end{abstract}

\section{Introduction}

A simple grid with a regular mesh placed normal to a steady uniform stream is the classic laboratory setup to study near-isotropic fluid turbulence (see e.g., Batchelor and Townsend 1948; Comte-Bellot and Corrsin 1966; Frenkiel et al. 1979; Kurian and Fransson 2009). Such an arrangement produces a turbulence which is homogeneous over cross-sections normal to the mean flow, but decays in the streamwise direction sufficiently far downstream of the

Electronic supplementary material The online version of this article (doi:10.1007/s00348-011-1070-x) contains supplementary material, which is available to authorized users.

R. Vonlanthen · P. A. Monkewitz ( $₫)$

Swiss Federal Institute of Technology Lausanne (EPFL),

CH-1015, Lausanne, Switzerland

e-mail: peter.monkewitz@epfl.ch grid where turbulence production is zero. However, without a carefully designed working section streamwise and cross-stream turbulence, intensities are unequal which is inherent to turbulence generated with a standard grid (Batchelor 1953; Comte-Bellot and Corrsin 1966; Uberoi and Wallis 1966). It was recently demonstrated (Lavoie et al 2007; Antonia et al. 2010) that a thin wire wrapped helically around the grid bars in conjunction with a small contraction after the grid produces nearly perfect isotropy.

There remains however the problem that standard grid turbulence is in most facilities limited to small Reynolds numbers $\operatorname{Re}_{\lambda}\left(=\left\langle u_{1}^{2}\right\rangle^{1 / 2} \lambda / v\right)$, based on the streamwise turbulence rms and the Taylor microscale, where the inertial range of turbulence length scales is poorly developed (Batchelor 1953). Here and in the following, the notation

$\widehat{u}_{i}=U_{i}+u_{i}$

for the decomposition of total velocities $\widehat{u}_{i}$ into time-mean $U_{i} \equiv\left\langle\widehat{u}_{i}\right\rangle$ and fluctuating components $u_{i}$ is chosen. High Reynolds numbers usually require very large facilities and/ or a high mean flow velocity, see e.g., Kistler and Vrebalovich (1966). Such conditions are difficult and expensive to realize, especially in hydraulic tunnels. To remedy this situation, a variety of sophisticated turbulence grids producing higher Reynolds numbers in 'normal' size facilities has been introduced in the past: Uberoi and Wallis (1966) increased the turbulence intensity with a honeycomb like grid; Ling and Wan (1972) developed a grid composed of vertical rods with horizontal oscillating agitators to intensify the turbulence; Mathieu and Alcaraz (1965), and later Gad-el-Hak and Corrsin (1974) increased the intensity with a 'jet grid' consisting of an array of vertical pipes injecting a secondary air flow into the mean stream. A remarkable feat was accomplished by Makita (1991) who designed an active grid with randomly rotating horizontal and vertical 
rods equipped with winglets. With such a device, Kang et al. (2003) obtained $\mathrm{Re}_{\lambda}$ 's similar to Kistler and Vrebalovich (1966), i.e., $\operatorname{Re}_{\lambda} \sim 700$, with a working section roughly ten times smaller than theirs. More recently, comparable Reynolds numbers have been realized (Seoud and Vassilicos 2007) with passive fractal grids developed by Hurst and Vassilicos (2007).

Unfortunately, none of the turbulence-intensifying grids mentioned in the previous paragraph improve isotropy relative to the basic square mesh grid, and in some cases, the deficit between streamwise and transverse intensities is even increased. Furthermore, the mechanical complexity of many of these grids makes them unsuitable for small facilities or liquid flows. This motivated the design of the present simple passive grid for an application in a relatively small water tunnel. The basic idea was to start from a plain square mesh grid and enhance the extraction of energy from the mean flow by appendices attached to the nodes of the grid. First attempts with flapping ribbons and flexible tubes were inconclusive. Then, inspired by the recent experimental and numerical investigations of the vigorous vortex-induced motion of tethered spheres in a steady stream (Williamson 1997; Govardhan and Williamson 2005; Lee et al, 2007), both leaden and buoyant spheres of different diameters were tested in our water tunnel. While the effect of the leaden spheres was unsatisfactory, the largest buoyant beads produced a marked increase in turbulence intensity and at the same time reduced anisotropy. In the following, the discussion is therefore restricted to grids with tethered wooden beads of different diameters for which the streamwise decay of turbulence intensity as well as streamwise velocity spectra is reported.

\section{Experimental method}

The new turbulence generators are based on a grid with square meshes of size $M=16 \mathrm{~mm}$ built of round stainless steel rods with a diameter $d=3 \mathrm{~mm}$ corresponding to a solidity of $34 \%$. Its overall size is $150 \times 200 \mathrm{~mm}^{2}$ with 8 by 11 complete meshes surrounded by partial peripheral meshes of $16 \times 11$ and $16 \times 12 \mathrm{~mm}^{2}$ (open areas of $13 \times 9.5$ and $13 \times 10.5 \mathrm{~mm}^{2}$ ), respectively. Wooden spherical beads are attached to the grid nodes by means of flexible silicone tubes with an outer diameter of less than $1 \mathrm{~mm}$. Two different tether lengths, $L_{1} \approx 1.4 M$ and $L_{2} \approx$ $M$, are used in a staggered arrangement to position the spheres in a region with strong mean flow gradients. A schematic of such a grid is shown in Fig. 1, which also defines the geometric parameters. Table 1 summarizes their values for the reference 'regular turbulence grid' without appendices (abbreviated RTG), the best 'enhanced turbulence grid' (ETG) with beads of $D=12 \mathrm{~mm}$ on all

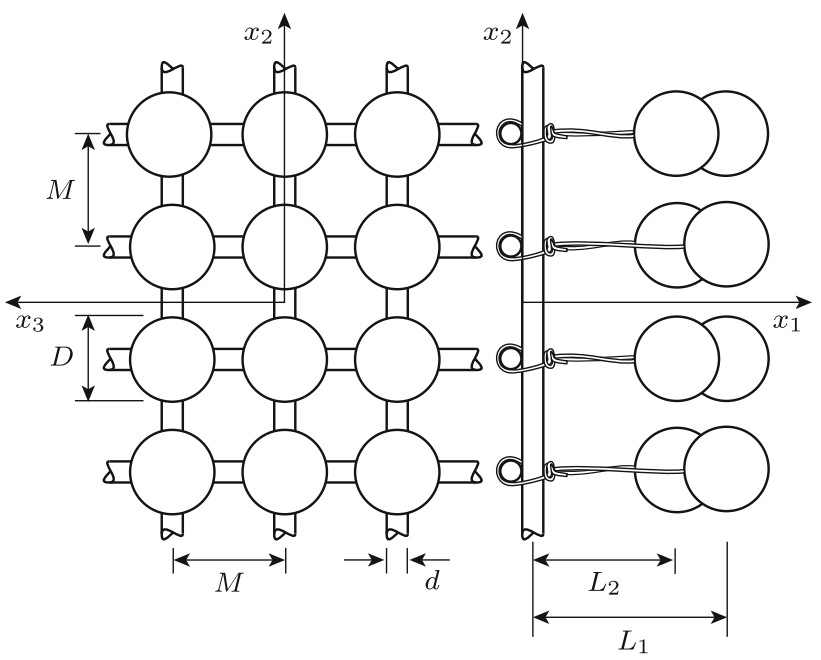

Fig. 1 Front and side view of part of the new grid with tethered beads and definition of geometrical parameters

Table 1 Parameters of the new turbulence generators: bead diameters, $D$, and densities, $\rho_{\text {bead }}$, are given relative to $M=16 \mathrm{~mm}$ and $\rho=998 \mathrm{~kg} \mathrm{~m}^{-3}$, respectively

\begin{tabular}{llllll}
\hline Grid & $D / M$ & $\rho_{\text {bead }} / \rho$ & Occupancy $(\%)$ & Bead solidity & Symbol \\
\hline RTG & - & - & - & - & $\square$ \\
ETG & 0.75 & 0.7 & 100 & 0.41 & $\bigcirc$ \\
D12 & 0.75 & 0.7 & 65 & 0.26 & $\star$ \\
D8 & 0.50 & 0.7 & 65 & 0.12 & $\nabla$ \\
D6 & 0.37 & 0.7 & 65 & 0.07 & $\diamond$ \\
\hline
\end{tabular}

nodes including peripheral nodes, and the three grids D12, D8, and D6 with beads of $D=12,8$ and $6 \mathrm{~mm}$, respectively. These last three grids which have no beads attached to peripheral nodes (equivalent to $65 \%$ node occupancy) are included because it was not a priori clear how to deal with peripheral nodes where wall interference potentially changes the effect of the beads. Note that on internal nodes, each bead is associated with $169 \mathrm{~mm}^{2}$ of open area, while the corresponding open area for peripheral beads is larger (208 $\mathrm{mm}^{2}$ on the sides and $221 \mathrm{~mm}^{2}$ on top and bottom) to accommodate the wall boundary layers. Table 1 also indicates the blockage ratio of the tethered spheres, which is the total projected bead area divided by the facility crosssection. For illustration, Fig. 2 shows a snapshot of the ETG in the running water tunnel. The trajectories of a smaller array of beads were tracked with a high-speed camera and were found to be highly irregular although the frequency spectrum is dominated by a strong Strouhal peak.

The experiments were conducted in a closed-loop water tunnel described in detail in Vonlanthen (2010). It has a 1-m-long straight working section with a rectangular 


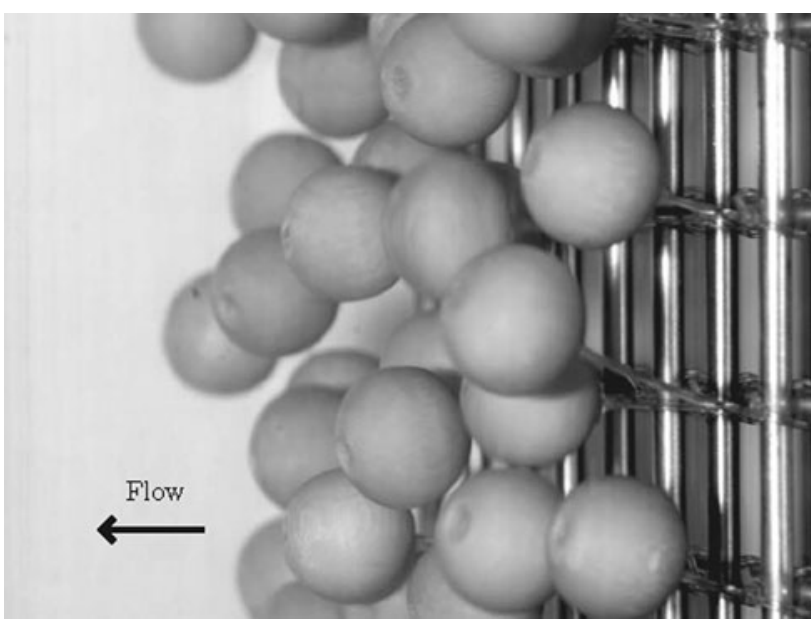

Fig. 2 Snapshot of the ETG mounted in the flow tunnel at $U_{0}=1.2 \mathrm{~m} \mathrm{~s}^{-1}$ (Movie online)

cross-section of $0.15 \mathrm{~m}$ by $0.20 \mathrm{~m}$, which accommodates the grids at its entry. The mesh size is at most as large as a tenth of the minimum side length and, therefore, the growth of the turbulence eddies should be unperturbed (Roach 1987). The mean flow velocity $U_{0}$, which is equal to the volume flow rate divided by the cross-section area, was held constant for all experiments at $1.20 \pm 0.05 \mathrm{~m} \mathrm{~s}^{-1}$ by means of a disk pump. This leads to a grid mesh based Reynolds number $U_{0} M / v$ of approximately $2 \times 10^{4}$. The working fluid was filtered tap water at room temperature, which remained constant to within $0.2^{\circ} \mathrm{C}$ during a measurement run. Its viscosity varied by less than $4.5 \%$ between the data sets, and the viscosities given in Table 2 are averages. Without the turbulence grids, the rms velocity fluctuations were less than $1 \%$ of $U_{0}$ over the central $60 \%$ of test section width and height. With the grids installed, the wall boundary layer displacement thickness at $x_{1} / M=20$ was estimated to be around $4 \mathrm{~mm}$ and, based on the increase in the mean velocity $U_{1}$ on the centerline, was found to grow by roughly $4 \%$ up to the end of the working section at 60 mesh widths from the grid. The wakes of the grid bars became undetectable at $x_{1} / M \geqslant 15$ for all grids, and at $x_{1} / M=20$ the deviations from transverse homogeneity of the turbulence intensities were within $0.5 \%$ for both the RTG and ETG. The other grids produced turbulence of comparable homogeneity, but in a smaller portion of the cross-section, i.e., $40 \%$ in width and height for the D12 at $x / M=20$. This is due to the missing peripheral spheres of the grids D6, D8, and D12. However, the turbulence production $-\left\langle u_{i} u_{j}\right\rangle \partial U_{i} / \partial x_{j}$ on the centerline was negligible for all grids (Vonlanthen 2010).

Velocities were measured along the streamwise centerline with a two-component Laser Doppler Anemometer (LDA, Dantec Fibre Flow) system and a single hot-film (HFA, TSI IFA 100) for streamwise velocity. Their signals served to determine both the turbulence intensities, $\left\langle u_{i} u_{i}\right\rangle$ as well as the longitudinal and cross-stream one-dimensional spectra $\phi_{i i}\left(\kappa_{1}\right)$, where $\kappa_{1}$ is the streamwise wave number component. The analog HFA signal was low-pass filtered at $0.1 \mathrm{~Hz}$ and high-pass filtered with a cutoff frequency well above the Kolmogorov frequency $\left(=U_{0} /(2 \pi \eta)\right.$, where $\eta$ is the Kolmogorov microscale) of $2 \mathrm{kHz}$. The filtered signal was digitized at a sampling rate of $20 \mathrm{kHz}$, and the calculated spectra had a frequency resolution of $0.1 \mathrm{~Hz}$, which allowed to resolve both ends of the turbulence spectrum including the most energetic scales with frequencies of $O(10 \mathrm{~Hz}$ ) (Wang and George 2002). Because of the insufficient spatial resolution due to the film length of $1 \mathrm{~mm}$, spectral corrections were applied according to Wyngaard (1968).

The LDA was operated in burst mode leading to irregular sampling with average data rates ranging from 0.6 to $2.5 \mathrm{kHz}$, depending on whether rms values or spectral samples were acquired. In burst mode (Buchave et al. 1979), the measured velocities are single realizations, rather than values averaged over multiple tracer particles in the measurement volume, which is $118 \mu \mathrm{m}$ in diameter and $3.3 \mathrm{~mm}$ long. Therefore, no corrections for spatial resolution had to be applied. However, because of a bias toward higher velocities, transit time weighting was used for rms velocities. Velocity spectra were then obtained by means of resampling the data and correcting the spectra for resampling noise and filter response (Simon and Fitzpatrick 2004).

Table 2 Streamwise TKE decay parameters of (2) and turbulence properties evaluated at $x_{1} / M=30$; the decay parameters $A$ and $n$ are given with $95 \%$ confidence intervals

\begin{tabular}{|c|c|c|c|c|c|c|c|c|c|c|c|c|}
\hline Grid & $v\left(\mathrm{~mm}^{2} \mathrm{~s}^{-1}\right)$ & $C_{\mathrm{p}}$ & $x_{\mathrm{vo}} / M$ & $A$ & $n$ & $\operatorname{Re}_{\lambda}$ & $\epsilon\left(\mathrm{cm}^{2} \mathrm{~s}^{-3}\right)$ & $\epsilon_{\phi}\left(\mathrm{cm}^{2} \mathrm{~s}^{-3}\right)$ & $\eta(\mathrm{mm})$ & $\lambda(\mathrm{mm})$ & $\Lambda_{1}(\mathrm{~mm})$ & $\overline{\Lambda_{2}(\mathrm{~mm})}$ \\
\hline RTG & 0.937 & 0.47 & 2.0 & $8.59 \pm 0.99$ & $1.22 \pm 0.04$ & 56 & 47.0 & 29.9 & 0.115 & 1.69 & 15.2 & 6.8 \\
\hline ETG & 0.941 & 1.02 & 3.0 & $3.18 \pm 0.10$ & $1.18 \pm 0.01$ & 100 & 151.9 & 88.5 & 0.086 & 1.69 & 13.3 & 6.2 \\
\hline D12 & 0.918 & 0.71 & 2.5 & $3.28 \pm 0.21$ & $1.24 \pm 0.02$ & 88 & 121.9 & 57.7 & 0.089 & 1.64 & 12.8 & 5.6 \\
\hline D8 & 0.918 & 0.57 & 3.5 & $7.83 \pm 0.31$ & $1.21 \pm 0.02$ & 61 & 59.7 & 32.0 & 0.107 & 1.64 & 10.1 & 3.8 \\
\hline D6 & 0.918 & 0.52 & 1.0 & $5.29 \pm 0.45$ & $1.31 \pm 0.03$ & 60 & 56.0 & 34.2 & 0.108 & 1.65 & 9.6 & 4.2 \\
\hline
\end{tabular}

The dissipation rates $\epsilon$ and $\epsilon_{\phi}$ were determined from streamwise turbulent energy decay and the spectra, respectively 
In addition, wall pressure taps were located at the entry of the working section $60 \mathrm{~mm}$ upstream of the grids, and a Pitot-static tube was placed $176 \mathrm{~mm}$, i.e., $11 \mathrm{M}$ downstream of the grid, providing the pressure drop coefficient $C_{\mathrm{p}} \equiv$ $2 \Delta p /\left(\rho U_{0}^{2}\right)$ across the grids. More detailed descriptions of the data processing for LDA, HFA and pressure measurements are given in Vonlanthen (2010).

\section{Results and discussion}

\subsection{Streamwise TKE decay}

The streamwise decay of the turbulence kinetic energy (TKE) defined as $k \equiv \frac{1}{2}\left\langle u_{i} u_{i}\right\rangle=\frac{3}{2} u^{2}$, with $u^{2} \equiv \frac{1}{3}\left\langle u_{i} u_{i}\right\rangle \approx$ $\frac{1}{3}\left(\left\langle u_{1}^{2}\right\rangle+2\left\langle u_{2}^{2}\right\rangle\right)$, is shown in Fig. 3 for all tested grids. The data are normalized as $3 u^{2} /\left(U_{0}^{2} C_{\mathrm{p}}\right)$ which corresponds to twice the turbulence kinetic energy per unit $C_{\mathrm{p}}$. Following Comte-Bellot and Corrsin (1966) each data set was individually fitted with the power law

$\frac{3 u^{2}}{U_{0}^{2}}=\frac{1}{A}\left(\frac{x_{1}-x_{\mathrm{vo}}}{M}\right)^{-n} \cong \frac{2\left(x_{1}-x_{\mathrm{vo}}\right) \epsilon}{U_{0}^{3} n}$

which is directly related to the dissipation rate $\epsilon$. Here, the origin of $x_{1}$ is always located in the plane between the vertical and horizontal grid bars, whereas the virtual origin of the turbulence decay $x_{\mathrm{vo}}$ can be on either side of the grid (Skrbek and Stalp 2000). Note that $x_{\mathrm{vo}}$ also incorporates any shift due to grid appendices. It is well known that the estimation of the decay exponent $n$ is delicate for measurements with limited streamwise extent (see for instance

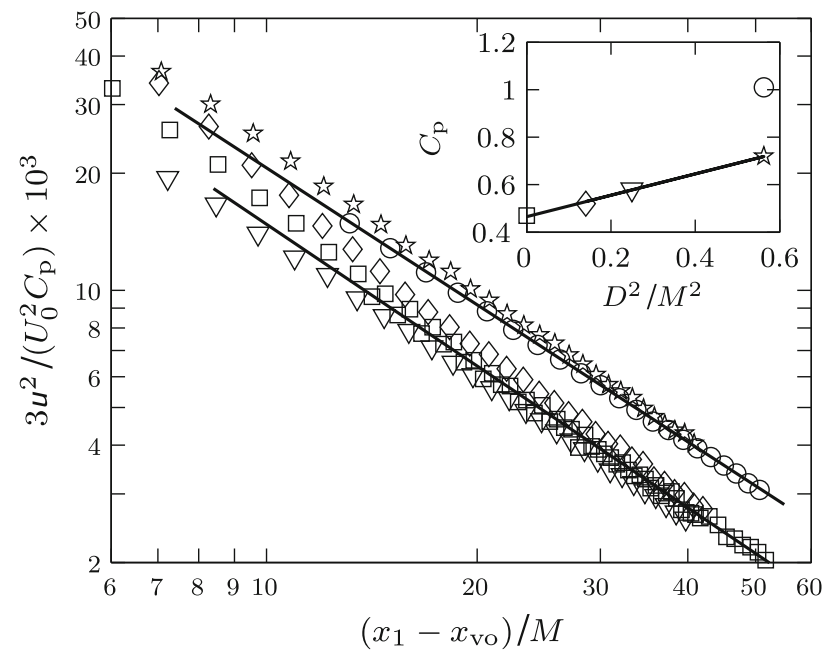

Fig. 3 Streamwise decay of TKE: solid line decay laws (2) of ETG and RTG. Inset pressure drop coefficient against the relative area blockage by the beads; solid line linear increase for $65 \%$ node occupancy. See Table 1 for symbols
Antonia et al, 2003). Therefore, we used the method described in detail by Lavoie et al. (2007), which was derived from the original procedure by Mohamed and LaRue (1990), to determine the decay parameters $A, x_{\mathrm{vo}}$ and $n$ with minimal ambiguity. In short, the method consists of varying $x_{\mathrm{vo}}$ and identifying the resulting power law region until the ambiguity of $A$ and $n$ is minimal. In all cases, the power law region was found to start at $x_{1} \approx 20$ $M$, which is plausible, as this corresponds to about twice the smallest test section dimension, which approximately defines the largest eddy size.

The fitted decay parameters, together with pressure drop coefficients used to scale the data in Fig. 3, are listed in Table 2 and are in fair agreement with literature values (see compiled data in e.g., Gad-el-Hak and Corrsin 1974; Mohamed and LaRue 1990). Note however that the values of $A$ and $x_{\mathrm{vo}}$ for the D8 grid do not fall between the D6 and D12 grids and illustrate the difficulty in fitting (2) over a short streamwise extent. The value of $n$ for the D8 grid, on the other hand, is in line with the other grids. The observed variation of $A$ and $n$ reflects, according to (George 1992), the dependence of the turbulence decay on initial conditions and is consistent with the findings by Lavoie et al. (2007). Specifically, $x_{\mathrm{vo}}$ is not equal to zero for the present square mesh biplane grids as was suggested by Mohamed and LaRue (1990), and the variation in grid geometry leads to (significant) changes in $n$. Finally, to investigate the robustness of the decay parameters in (2), we also fitted the same data with $x_{\mathrm{vo}}=0$ and found $A$ 's and $n$ 's that differ from the values given in Table 2 by as much as 50 and $10 \%$, respectively. However, the resulting estimates of $\epsilon$ changed by less than $5 \%$, indicating that the fits with and without virtual origin were of equal quality over our limited streamwise interval (by definition, any good fit of the measured $u^{2}\left(x_{1}\right)$ must yield, within experimental error, the same $\epsilon$.)

Figure 3 shows the decay laws (2) for the RTG and the ETG as the two solid lines. Two distinct groups of data are evident, with the upper group corresponding to the two grids with the largest beads, i.e., to the ETG and the D12, and the lower group including all the other grids. According to this plot, grids with beads of a diameter close to the mesh size produce more turbulence than a plain grid with the same pressure drop. The latter appears to grow linearly with the bead diameter squared, as shown by the inset in Fig. 3. The larger $C_{\mathrm{p}}$ for the ETG is due to the $100 \%$ node occupancy, where all peripheral nodes are also equipped with tethered spheres. Smaller beads are seen to offer no advantage as they produce nearly the same TKE per unit $C_{\mathrm{p}}$ as the plain reference grid RTG.

In classical grid turbulence, it was observed that at high blockage ratios the jets emerging from the grid meshes 
coalesce to produce large-scale velocity inhomogeneities. This phenomenon was not observed with the new beaded grids as the ETG's closed-area ratio of 0.41 is below the stability limit of 0.43 (Bradshaw 1965). More importantly, the moving beads produce jets that move around on time scales which do not allow the slow collective jet interactions to develop. Of particular, interest is the doubling of the TKE per unit $C_{\mathrm{p}}$ for the two grids with large beads relative to the other grids. One might think of an explanation with a straightforward momentum control volume extending from upstream of the grid to a downstream section where $U_{0}$ is again spatially uniform. However, the result $3 u^{2} /\left(U_{0}^{2} C_{\mathrm{p}}\right)=(3 / 2)\left[1+\left(C_{\mathrm{d}} / C_{\mathrm{p}}\right)\right]>3 / 2$, with $C_{\mathrm{d}}$ being the drag coefficient of the grid, is clearly in contradiction with Fig. 3. The reason is that the dissipation between the grid and the downstream face of the control volume has not been taken into account. Hence, we propose a more subtle explanation of the doubling of TKE per unit $C_{\mathrm{p}}$ for the two grids with large beads, namely a transfer of mean to turbulent momentum flux downstream of the grid by enhanced random motion of the grid bar wakes. The following simple one-dimensional example may explain the idea better than words: Choosing the total $x_{1}$ velocity just downstream of the grid as

$\widehat{u}_{1}\left(x_{2}, t\right)=1+\alpha \cos \left[\beta x_{2}-\beta f(t)\right]$,

with $\beta=2 \pi / M$ and $f(t)$ a random function with zero mean, yields the time-mean velocity

$U_{1}\left(x_{2}\right)=1+\alpha \cos \left(\beta x_{2}\right)\langle\cos (\beta f)\rangle$

and the $x_{2}$-dependent streamwise turbulence intensity

$$
\begin{aligned}
\left\langle u_{1}^{2}\right\rangle= & \alpha^{2} \cos ^{2}\left(\beta x_{2}\right)\left[\left\langle\cos ^{2}(\beta f)\right\rangle-\langle\cos (\beta f)\rangle^{2}\right] \\
& +\alpha^{2} \sin ^{2}\left(\beta x_{2}\right)\left\langle\sin ^{2}(\beta f)\right\rangle .
\end{aligned}
$$

Averaging over $x_{2}$, indicated by an over-bar, yields a constant total average momentum flux per unit area of $\overline{U_{1}^{2}+\left\langle u_{1}^{2}\right\rangle}=1+\left(\alpha^{2} / 2\right)$ with a variable turbulence part $\overline{\left\langle u_{1}^{2}\right\rangle}=\left(\alpha^{2} / 2\right)\left[1-\langle\cos (\beta f)\rangle^{2}\right]$ depending on the function $f(t)$. Hence, if the lateral positions of grid bar wakes can be randomized with sufficient amplitude, a significant portion of the total momentum flux can be shifted to turbulence. We submit that in our setup, only the largest beads with a diameter comparable to $M$ do this without too much drag penalty.

Accepting the premise of isotropy, turbulence energy dissipation rates, $\mathrm{d} k / \mathrm{d} t=-\epsilon \approx U_{0} \mathrm{~d} k / \mathrm{d} x_{1}$, Kolmogorov microscales, $\eta \equiv\left(v^{3} / \epsilon\right)^{1 / 4}$, Taylor microscales, $\lambda^{2} \equiv 15 v u^{2} / \epsilon$, and Reynolds numbers, $\operatorname{Re}_{\lambda} \equiv u \lambda / v$, were derived from (2) (see e.g., Tennekes and Lumley 1972) and are also specified in Table 2. The resulting values for $\operatorname{Re}_{\lambda}$ are typical for laboratory turbulence in smaller facilities (c.f. compilation by

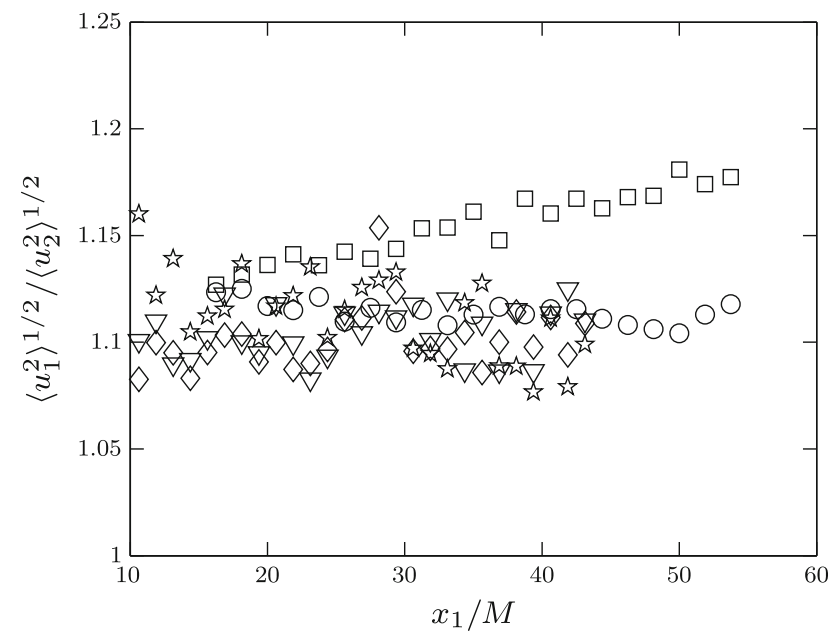

Fig. 4 Streamwise evolution of the overall turbulence anisotropy $\left\langle u_{1}^{2}\right\rangle^{1 / 2} /\left\langle u_{2}^{2}\right\rangle^{1 / 2}$. See Table 1 for symbols

Makita 1991) but are well below the largest $\operatorname{Re}_{\lambda}$ achieved with active (Kang et al. 2003) or fractal grids (Seoud and Vassilicos 2007). Nevertheless, Table 2 demonstrates that our best grid is capable of approximately doubling $\operatorname{Re}_{\lambda}$ by doubling the turbulence intensity while leaving the Taylor microscale virtually unaffected.

In comparison with the RTG, a modest improvement of the overall isotropy $\left\langle u_{1}^{2}\right\rangle^{1 / 2} /\left\langle u_{2}^{2}\right\rangle^{1 / 2}$ is achieved with all beaded grids, as seen in Fig. 4. In particular, the anisotropy in Fig. 4 for the beaded grids remains at a constant level, while for the RTG, the discrepancy between streamwise and transverse intensities grows with downstream distance; recall that no attempt has been made to improve isotropy with a slight contraction after the grid (Comte-Bellot and Corrsin 1966). A possible explanation for the slow increase in anisotropy behind the RTG is the longer persistence of the grid bar wakes, which are not broken up by moving spheres (see e.g., Lavoie et al. 2007). Their flapping is thought to contribute predominantly low frequency to $\left\langle u_{1}^{2}\right\rangle$. Their long persistence, on the other hand, allows the von Kármán wake instability to develop fully and contribute mainly higher-frequency turbulence to $\left\langle u_{1}^{2}\right\rangle$ which decays faster with downstream distance. This is consistent with the view that the level of anisotropy is strongly dependent on the initial conditions and is dominated by large scales (e.g., Batchelor and Stewart 1950) as seen also in the spectra to be discussed next.

\subsection{Velocity spectra}

Velocity spectra were measured with LDA and HFA at 30 mesh widths downstream of the grid and are shown in Fig. 5 in Kolmogorov variables. The inertial spectra $\alpha_{i} \epsilon^{2 / 3} \kappa_{1}^{-5 / 3}$ are indicated by the dashed lines, with 

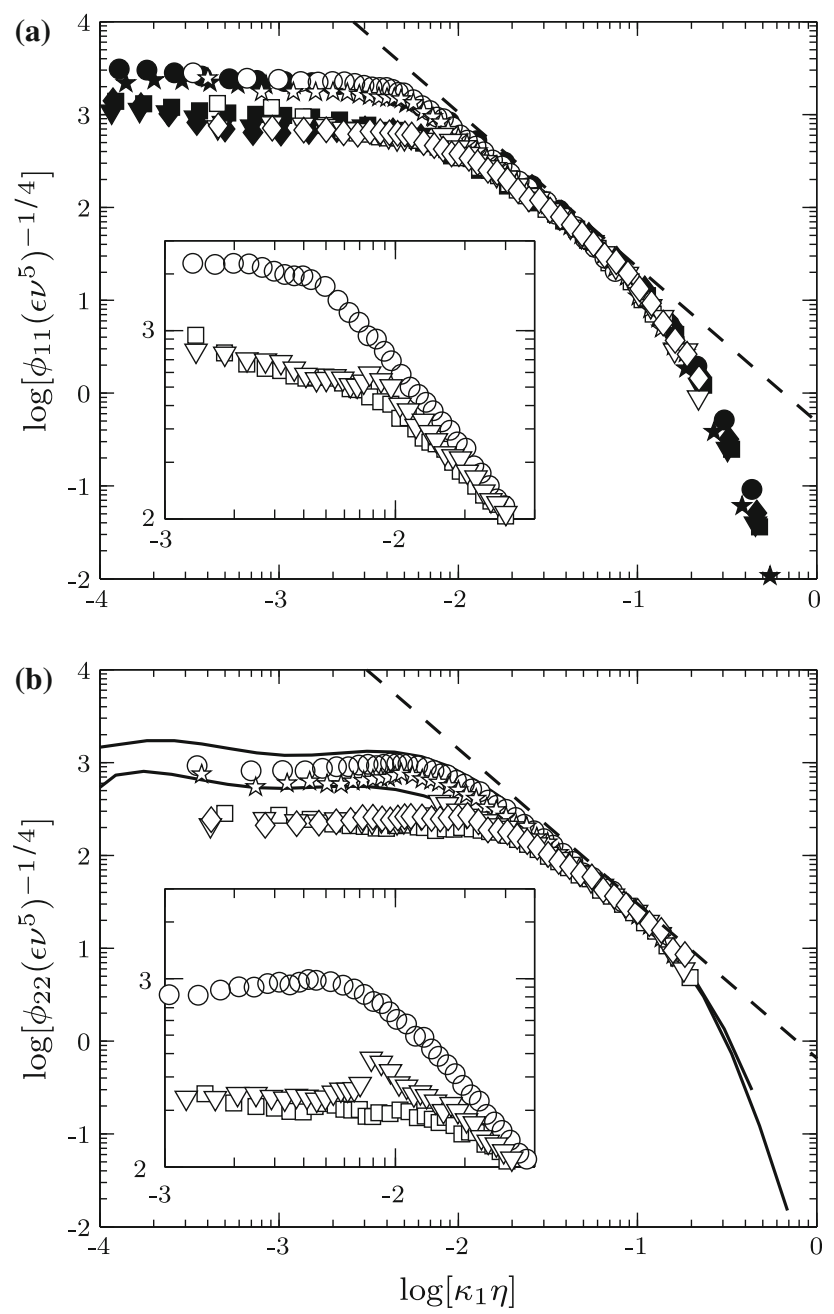

Fig. 5 a Streamwise and b transverse spectra measured with LDA (open symbols) and HFA (solid symbols): dashed line Kolmogorov spectrum; solid line isotropic spectra obtained from HFA measurements for the ETG and the RTG. Insets close-ups of the RTG, ETG and D8 grid. See Table 1 for symbols

$\alpha_{1}=\frac{3}{4} \alpha_{2}=\frac{18}{55} 1.5$ being the Kolmogorov constants. Performing the integral $15 v \int_{0}^{\infty} \kappa_{1}^{2} \phi_{11} \mathrm{~d} \kappa_{1}$ yields estimates $\epsilon_{\phi}$ of the dissipation rate, which are seen in Table 2 to be between 36 and 53\% lower than the corresponding $\epsilon$ determined from the power law. Since $\left\langle u_{i}^{2}\right\rangle=\int_{0}^{\infty} \phi_{i i} \mathrm{~d} \kappa_{1}$ is verified, there is no obvious explanation for the difference between $\epsilon$ and $\epsilon_{\phi}$ and no spectral measurements at other streamwise locations are available to help explaining it. We only note here that Kurian and Fransson (2009) also report comparable differences of up to $50 \%$ and suggest that they are a consequence of using an isotropic formalism to extract $\epsilon$ from anisotropic turbulence. We also note here in passing that intermittency (see for instance Skrbek and Stalp 2000), by attenuating the high wave number end of the derivative spectrum, could also contribute to the discrepancy between $\epsilon$ and $\epsilon_{\phi}$ but is unlikely to explain the whole difference.

The overall collapse for the different grids at high wave numbers seen in Fig. 5 verifies spectral universality at small turbulence scales. Furthermore, for all grids, the turbulence is locally isotropic. That is to say, the turbulence is isotropic in the (short) inertial range and beyond, as evidenced by the agreement between the measured transverse spectra, $\phi_{22}$, and the isotropic spectra, $\phi_{22}=\frac{1}{2}\left[\phi_{11}-\kappa_{1} \partial \phi_{11} / \partial \kappa_{1}\right]$ computed from the HFA data for the ETG and the RTG (the two solid lines in Fig. 5b). Similar to the streamwise energy decay, the spectra form two groups, one with the RTG and the grids with smaller beads and another one with ETG and D12 with increased energy in the large scales including the injection scale. One also observes that for these grids, ETG and D12, the measured lateral spectra in the low wave number region are closer to the theoretical isotropic spectra, which is the reason for the better rms-velocity ratio, $\left\langle u_{1}^{2}\right\rangle^{1 / 2} /\left\langle u_{2}^{2}\right\rangle^{1 / 2}$. In other words, the large beads introduce proportionally more low-frequency transverse velocity fluctuations. As speculated earlier, this may come about by the breakup of the long grid bar wakes which, by their undulations, tend to produce predominantly streamwise fluctuations. Furthermore, due to the larger energy injection by the ETG and D12 grids, the relatively small inertial range is extended to roughly one decade of $\kappa_{1} \eta$.

Considering that the motion of a single tethered sphere is often dominated by a single frequency, one may ask whether the spectra of beaded grids are contaminated by local peaks. The insets of Fig. 5 showing close-ups of the early inertial range reveal peaks for the $\mathrm{D} 8$ grid. These peaks correspond to the dominant frequency of the bead oscillations with a Strouhal number $f D / U_{0}$ estimated at 0.1 by means of a highspeed camera. No such peaks could be identified for the other grids. The likely reason is that the largest spheres generate turbulence in the same wave number band as the plain grid itself. It may also be that the motion of the large spheres is more broad band due to a stronger interaction with the grid wakes and/or collisions between the beads, but this line of investigation has not been pursued.

Finally, correlation functions were computed from the velocity spectra to determine the longitudinal and lateral integral scales, $\Lambda_{1}$ and $\Lambda_{2}$, of each grid (c.f. Table 2). These scales are seen to increase with growing sphere diameter, but the largest integral scales were obtained with the plain grid. The finding that $\Lambda_{1}$ is relatively close to the corresponding bead diameter suggests that the tethered spheres leave their mark on the turbulence characteristics such as the integral length scale. Furthermore, the ratio $\Lambda_{1} / \Lambda_{2}$ is closer to two for the ETG and the D12 grids than for the RTG, which is consistent with the observed improved turbulence isotropy. 


\section{Conclusion}

A new passive grid has been developed, which converts the energy of an oncoming uniform stream more efficiently into turbulence that is in addition more isotropic than turbulence produced by a plain grid. The new grid presents advantages over existing turbulence-enhancing grids such as simple design, low cost, and the possibility to use it in liquid or gas flow facilities of any size. Despite a limited optimization, a $\operatorname{Re}_{\lambda}$ roughly twice as large as with a conventional grid has been attained with spherical beads of diameter $D / M=0.75$. The performance can quite possibly be further improved with even larger beads, possibly not occupying every grid node, or beads of different nonspherical shapes.

Acknowledgments This work has benefitted from the financial support of Swiss National Research Fund (SNF) grant no. 200020-119824.

\section{References}

Antonia RA, Smalley RJ, Zhou T, Anselmet F, Danaila L (2003) Similarity of energy structure functions in decaying homogeneous isotropic turbulence. J Fluid Mech 487(1):245-269

Antonia RA, Lavoie P, Djenidi L, Benaissa A (2010) Effect of a small axisymmetric contraction on grid turbulence. Exp Fluids 49(1): 3-10

Batchelor GK (1953) The theory of homogeneous turbulence. Cambridge University Press, Cambridge (reprinted 1970)

Batchelor GK, Stewart RW (1950) Anisotropy of the spectrum of turbulence at small wave-numbers. Quart J Mech Appl Math $3(1): 1-8$

Batchelor GK, Townsend AA (1948) Decay of isotropic turbulence in the initial period. P Roy Soc Lond A Mat 193(1035):539-558

Bradshaw P (1965) The effect of wind-tunnel screens on nominally two-dimensional boundary layers. J Fluid Mech 22:679-687

Buchave P, George WKJ, Lumley JL (1979) The measurement of turbulence with the laser-Doppler anemometer. Annu Rev Fluid Mech 11:443-503

Comte-Bellot G, Corrsin S (1966) The use of a contraction to improve the isotropy of grid-generated turbulence. J Fluid Mech 25: 657-682

Frenkiel FN, Klebanoff PS, Huang TT (1979) Grid turbulence in air and water. Phys Fluids 22(9):1606-1617
Gad-el-Hak M, Corrsin S (1974) Measurements of the nearly isotropic turbulence behind a uniform jet grid. J Fluid Mech 62(01): $115-143$

George WK (1992) The decay of homogeneous isotropic turbulence. Phys Fluids A Fluid 4(7):1492-1509

Govardhan RN, Williamson CHK (2005) Vortex-induced vibrations of a sphere. J Fluid Mech 531:11-47

Hurst D, Vassilicos JC (2007) Scalings and decay of fractal-generated turbulence. Phys Fluids 19(3):035-103

Kang HS, Chester S, Meneveau C (2003) Decaying turbulence in an active-grid-generated flow and comparisons with large-eddy simulations. J Fluid Mech 480:129-160

Kistler A, Vrebalovich T (1966) Grid turbulence at large Reynolds numbers. J Fluid Mech 26:37-47

Kurian T, Fransson JHM (2009) Grid-generated turbulence revisited. Fluid Dyn Res 41(2):021-403

Lavoie P, Djenidi L, Antonia RA (2007) Effects of initial conditions in decaying turbulence generated by passive grids. J Fluid Mech 585(1):395-420

Lee H, Thompson MC, Hourigan K (2007) Vortex-induced vibration of a neutrally buoyant tethered sphere. In: 16th Australasian fluid mechanics conference

Ling SC, Wan CA (1972) Decay of isotropic turbulence generated by a mechanically agitated grids. Phys Fluids 15(8):1363-1369

Makita H (1991) Realization of a large-scale turbulence field in a small wind tunnel. Fluid Dyn Res 8:53-64

Mathieu J, Alcaraz E (1965) Réalisation dune soufflerie à haut niveau de turbulence. C R Acad Sci 261:2435

Mohamed MS, LaRue JC (1990) The decay power law in gridgenerated turbulence. J Fluid Mech 219:195-214

Roach P (1987) The generation of nearly isotropic turbulence by means of grids. Int J Heat Fluid Fl 8(2):82-92

Seoud RE, Vassilicos JC (2007) Dissipation and decay of fractalgenerated turbulence. Phys Fluids 19(10):105-108

Simon L, Fitzpatrick J (2004) An improved sample-and-hold reconstruction procedure for estimation of power spectra from LDA data. Exp Fluids 37(2):272-280

Skrbek L, Stalp SR (2000) On the decay of homogeneous isotropic turbulence. Phys Fluids 12(8):1997-2019

Tennekes H, Lumley JL (1972) A first course in turbulence. MIT Press, Cambridge

Uberoi M, Wallis S (1966) Small axisymetric contraction of grid turbulence. J Fluid Mech 24:539-543

Vonlanthen R (2010) The effects of fluid elasticity on grid turbulence. $\mathrm{PhD}$ thesis, EPFL

Wang H, George WK (2002) The integral scale in homogeneous isotropic turbulence. J Fluid Mech 459(1):429-443

Williamson C (1997) Advances in our understanding of vortex dynamics in bluff body wakes. J Wind Eng Ind Aerod 69(71): 3-32

Wyngaard JC (1968) Measurement of small-scale turbulence structure with hot wires. J Phys E Sci Instrum 1(11):1105-1108 